\title{
Application of Fault Diagnosis Technology in Special Vehicle Electrical System
}

\author{
WANG Kun ${ }^{1, \mathrm{a}}$, LIN Qing ${ }^{1, \mathrm{~b}}$, LI Shen ${ }^{1, \mathrm{c}}$, LIN Can ${ }^{1, \mathrm{~d}}$, WEI Min ${ }^{1, e}$ \\ ${ }^{1}$ China North Vehicle Research Institute, Beijing 100081, China

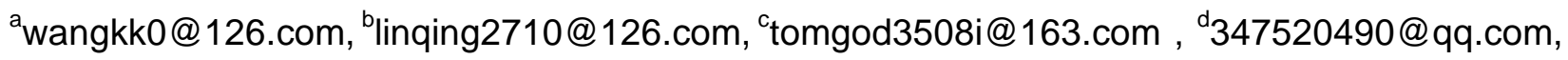 \\ e284093749@qq.com
}

Keywords: fault diagnosis technology;fault diagnosis;diagnosis equipments

Abstract : This paper introduces the application of fault diagnosis technology at home and abroad, and puts forward the way of applying the fault diagnosis technology of special vehicle electrical system. This method is conducive to improving the capabilities of vehicle electrical system fault diagnosis, and reduces the repair time of fault.

\section{Introduction}

With the continuous development of electronic technology, special vehicle electrical system has also experienced the process from simple to complex, from low level to high level. Especially in recent years, the requirement of vehicle on automatic control system have continuously improved, the electrical system undertakes more and more tasks,and its status is also increasingly important. In addition to the quality of supplied power, the reliability, maintainability and testability of the electrical system have become objectives that attract people's close attention ${ }^{[1]}$. Electrical system is the support system of the whole vehicle, if any fault in the electrical system can not be detected and eliminated in time, the fault may be further expanded, resulting in the changes of state of the upper system and the faulty functions, affecting the implementation of the task, and even threatening safety. Therefore, in order to accurately, reliably and quickly detect, isolate and eliminate faults, the fault diagnosis technology should be applied to the design of the electrical system to improve the fault diagnosis capability of the electrical system and reduce the vehicle maintenance time and ensure the combat effectiveness of the whole vehicle.

\section{Current situation of the application of fault diagnosis technology}

\section{Situation of application of foreign fault diagnosis technology}

Foreign fault diagnosis technology began at the mid-60s of last century, and has 40 years of history. Early in 1970s, as the increasing complexity of the equipment, the probability of failure caused by design, manufacture and maintenance problems increased, which caused the military of United States and Britain and other industrial sectors' attention. In order to solve the problem of poor testing equipment, the long time of fault diagnosis and high security costs, the US Air Force and the Navy have carried out research one after another, and have taken measures aiming at the diagnosis factors. The US Air Force developed the General Integrated Maintenance and Diagnostic System (GIMADS), and the US Navy developed the Integrated Diagnostic Support System (IDSS) which were used in the development of F-22 fighters and M1A2 main battle tanks. In the Iraq war, the US military's digital maintenance was also widely used, all the tanks in the third mechanized infantry division were equipped with a digital diagnosis and prediction PMA (Portale Maintenance Aids), with a reference of PMA regulations, it can conduct fault diagnosis and the necessary maintenance, and greatly shorten the fault repair time ${ }^{[2]}$. 


\section{Situation of application of domestic fault diagnosis technology}

Domestic fault diagnosis technology started later that foreign countries, in 1979 the initial contact with the equipment diagnostic technology was touched, compared with foreign countries, although the theoretical research in our countries has been closed to the world level, but there is still a large gap in application between our country and foreign countries. The aerospace industry has done a lot of work in this area, through the application of BIT (in-house test), interactive electronic manual, ATE (automatic test equipment) and other test methods to improve the level of automation of fault diagnosis and reduce the maintenance time of fault ${ }^{[3]}$. At present, the basic mode of troubleshooting of special vehicles is: to find fault $\rightarrow$ report to maintenance personnel $\rightarrow$ locate the fault $\rightarrow$ solve the problem, more often it depends on experienced technical staff to diagnose and eliminate the fault. It has a strong dependence on technical personnel if there is no complete fault Diagnostic system and automatic maintenance equipment.

\section{The situation of application of special vehicle electrical system fault diagnosis technology}

\section{Characteristics of electrical system fault diagnosis and existing problems}

Electrical system fault diagnosis generally use traditional diagnostic techniques, and is based on the detection of data processing diagnostic methods. After the fault happened in the test site, using a simple instrument to detect the signal, and then through the experienced engineering and technical personnel with their experience and knowledge to analysis and judge and identify the cause of the fault and the location of the fault, then the appropriate maintenance measures are taken. However, in the actual work, there are more failures, and the fault association is strong, the cause of the fault is very cumbersome, which brings considerable difficulties to the troubleshooting personnel. The following is the histogram of the statistics of fault mode and fault number of the sub-system of a large number of vehicles' electrical system. Figure 1 is the distribution column chart of the number of electrical system statistics failure, Figure 2 is the electrical system statistics fault type distribution column chart.

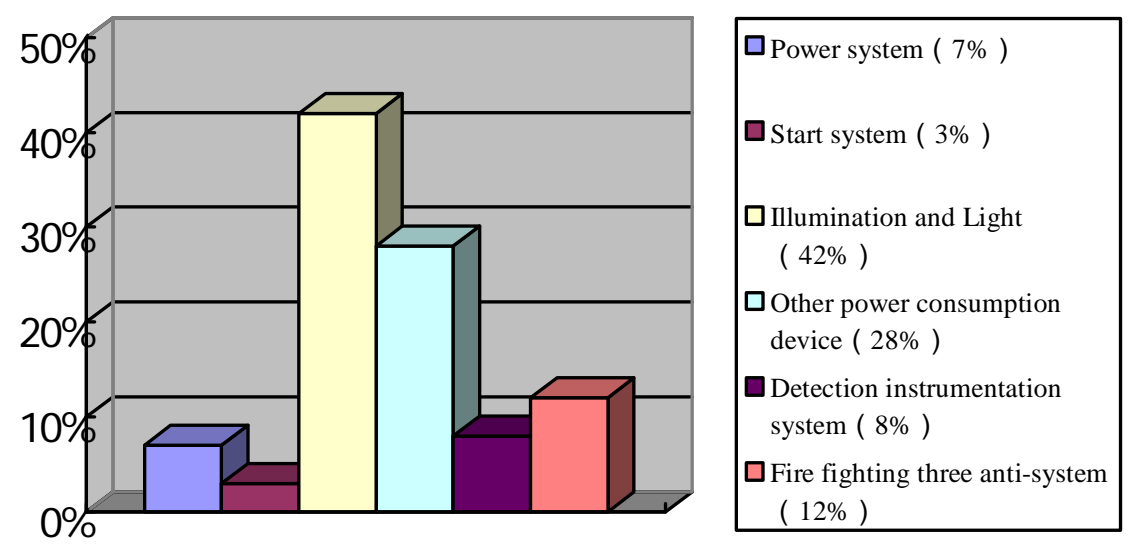

Fig. 1 Distribution column chart of the number of electrical system statistics 


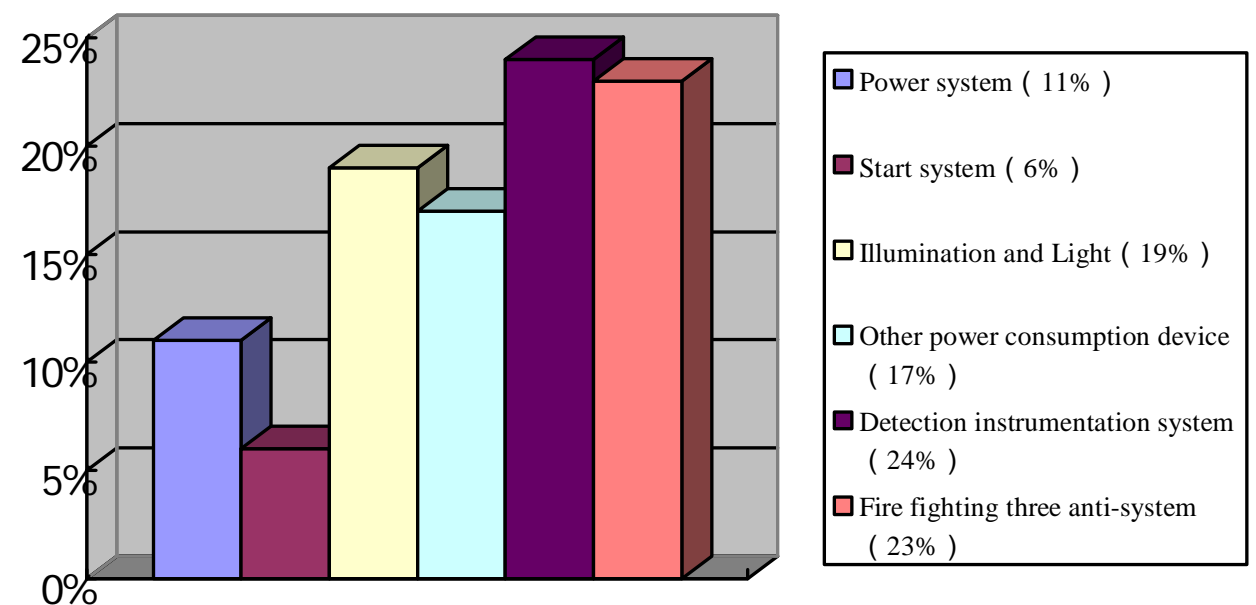

Fig. 2 Electrical system statistics fault type distribution column chart

It can be seen in the bar chart that there are many types and a large quantity of electrical system fault, only rely on the experience and the law of the summary,the theory that based on is fuzzy, incomplete, and background information is not clear, which is easy to result in the loss of knowledge of fault diagnosis, which greatly reduces the accuracy of the system and increase the time of troubleshooting. Therefore, the electrical system should change the traditional fault diagnosis mode, and adapt the advanced automated diagnostic equipment to improve the ability of fault diagnosis.

\section{The way of application of electrical system fault diagnosis technology}

Electrical system fault diagnosis should apply the way of combining automatic testing with manual testing method, and it is divided into field-level diagnosis and factory-level diagnosis, as shown in Figure 3:

Field level diagnosis
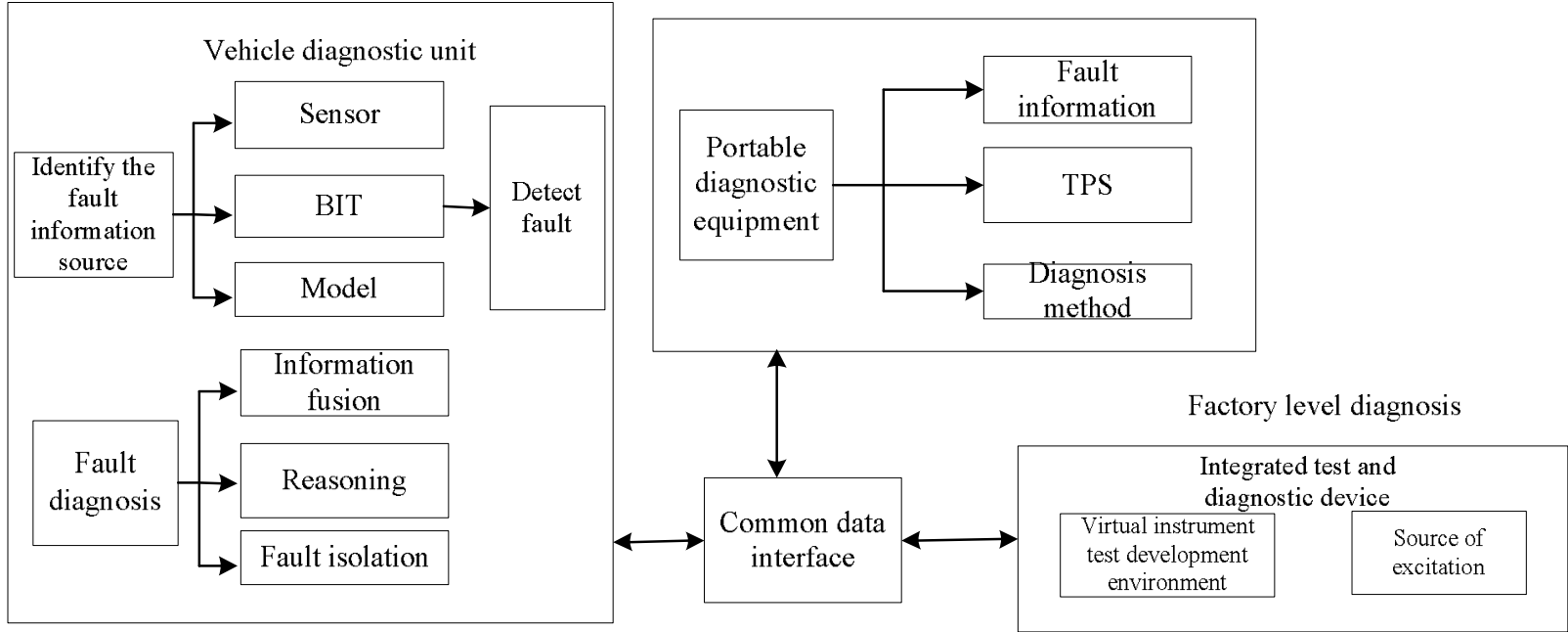

Fig. 3 Structural frame drawing of electrical system fault diagnosis

Field-level diagnosis mainly through the vehicle diagnostic system, portable diagnostic equipment to isolate the fault into the component level, that is, control box level; factory-level diagnosis main through a comprehensive test diagnostic equipment to complete the board-level or even component-level fault diagnosis. These diagnostic devices have a common data interface, so they can not only conduct real-time online diagnosis, but also can conduct off-line diagnosis to meet different needs, and to achieve $100 \%$ of the troubleshooting.

1) Vehicle diagnostic system

Vehicle fault diagnosis system uses distributed / centralized method, which are shown in Figure 4: 


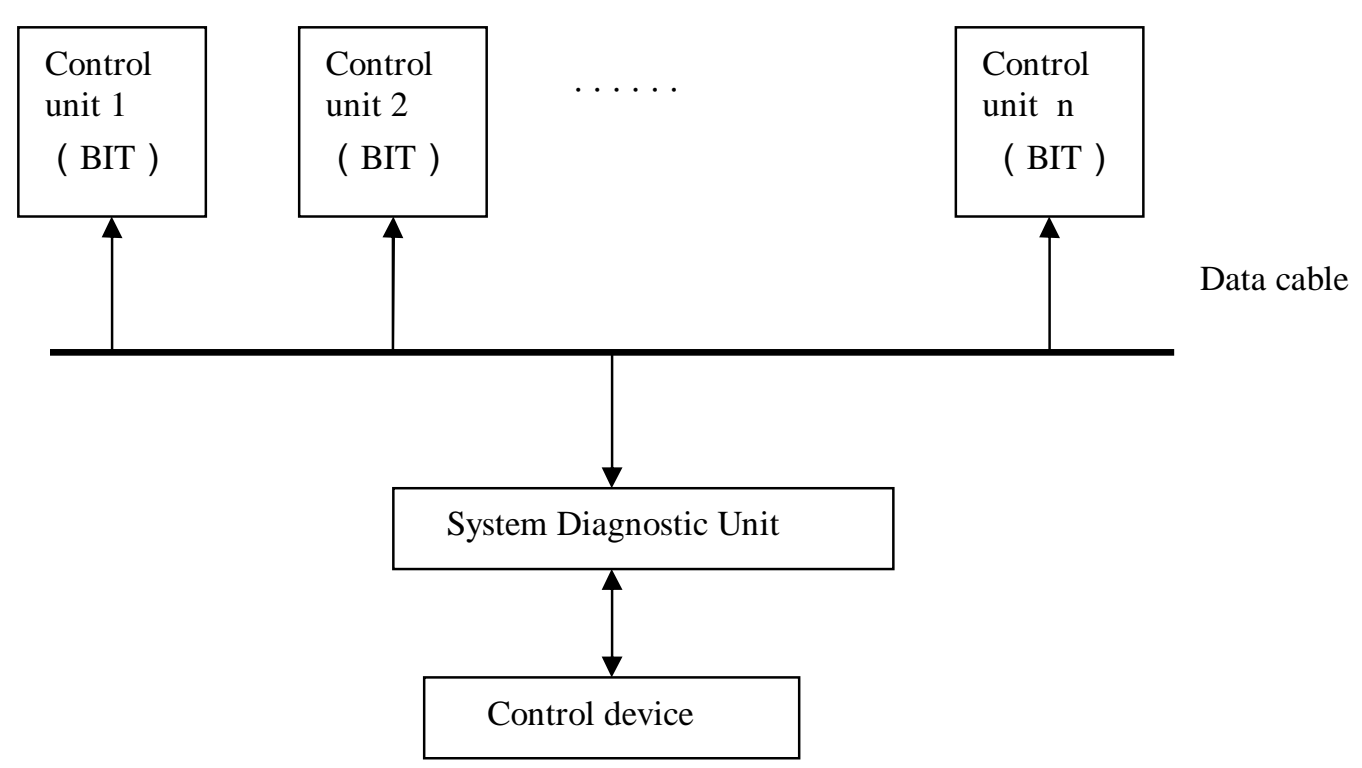

Fig. 4 Structural frame drawing of vehicle diagnostic system

The vehicle diagnostic system consists of two parts, one part is the component-level self-diagnosis, and the other part is system-level diagnosis (completed by the system diagnostic unit).

Component-level self-diagnosis requires that when in the design, each control components under the premise of completing their own functions, the components should also be added with testable design, and use embedded BIT technology to complete the self-testing, and can periodically transform the status of the information into fault code to upload (such as the input signal fault of control box, the output signal fault, etc.) to the system-level diagnostic unit.

After the system-level diagnostic unit receiving the fault code uploaded by the control unit, it can merge the fault information and comprehensively judge the fault through the internal reasoning mechanism and classify it. Part of the results are displayed to the driver, to assist the driver to analyze what kind of maintenance methods shoule be taken (parking maintenance, the use of degraded use function, etc.), and part of the results are converted into maintenance information to help maintenance staff to conduct component-level maintenance and replacement, the other part only play the role of recording to help design personnel find design deficiency and improve designs.

2) Portable diagnostic equipment

No diagnostic equipment can be perfect, the vechicle diagnostic unit can not complete all the diagnosis of fault correctly, portable diagnostic equipment is a diagnostic equipment used to make up for this deficiency.

It is easy to carry, through the way of fault pheninmen to find the cause of the fault, it supports the function of quickly querying path according to the priority, and assists maintenance personnel to do some routine checks. The built-in interactive electronic technical manual helps maintenance personnel on the analysis of routine maintenance, testing and maintenance, spare parts management and maintenance information. And it has a data interface with the vehicle diagnostic unit, which can download the stored data to the device, and on the site to monitor key components of the measured object to obtain detailed diagnostic data.

3) Comprehensive test and diagnosis equipment

The comprehensive test and diagnostic equipment is mainly used for the diagnosis of component-level faults. Through the virtual instrument test to develop environment, and to put the external excitation on the components or start the embedded BIT test and other methods. It can verify the fault and conduct fault isolation then quickly locate the smallest replaceable units. The diagnostic results, maintenance recommendations and component status of the wrong data are graphically displayed, to guide the maintenance staff maintain the board level and even component level. The 
comprehensive test diagnostic equipment also has the function of recording maintenance information, outputing maintenance reports, and analyzing historical data.

\section{Conclusions}

Fault diagnosis technology is developping as the modern equipment is more and more complex and the degree of automation is more and more higher. Compared with the traditional way, the way that adopted in the above fault diagnosis can effectively shorten the fault repair time, improve the degree of automation of fault diagnosis and reduce the dependence on professional and technical personnel to greatly meet the needs of future weapons and equipment. But to really apply these ways, there are still a lot of work to do, such as the reliability design of system and product, testability design, the construction ans selection of fault model library, these all have to be further identified and improved.

\section{References}

[1] Zeng Shengkui. Reliability Design and Analysis[M].Beijing: National Defense Industry Press,2011. [2] Tian Zhong, Shi Junyou. Analysis and verification of system test design [M]. Beijing: Aerospace University Press, 2003.

[3] Shi Junyou. Testability Design Analysis and Verification[M]. Beijing: National Defense Industry Press, 2011. 